\title{
Phylogenetic autocorrelation and evolutionary constraints in worker body size of some neotropical stingless bees (Hymenoptera: Apidae)
}

\author{
MARIA IZABEL BARNEZ PIGNATA \& JOSÉ ALEXANDRE FELIZOLA DINIZ-FILHO* \\ Departamento de Biologia Geral, Instituto de Ciências Biológicas, Universidade Federal de Goiás, CP 131, \\ 74.001-970, Goiânia, Goiás, Brazil
}

\begin{abstract}
Developments in the analysis of comparative data in evolutionary biology suggest that it is possible to partition the total variance of some quantitative trait into a phylogenetic component, which contains that part of the variation attributed to common ancestry with other species, and a specific component, which results from independent evolution after cladogenesis. In this paper, we analysed the variation in worker body size across 16 neotropical species of stingless bees (Meliponinae), using phylogenetic autocorrelation models to evaluate evolutionary constraints in this complex trait. Body size was defined in a multivariate sense as the first principal component extracted from the covariance matrix of 10 log-transformed morphometric characters. The estimated phylogenetic autoregressive coefficient $(p)$ was equal to $0.495 \pm 0.192$, indicating that only 24.5 per cent of variability in worker body size can be attributed to inertial phylogenetic effects. This relatively low value of $p$ in bees is to be expected, considering that this complex trait has traditionally been recognized as an expression of worker adaptation related to foraging activity and resource exploitation.
\end{abstract}

Keywords: body size, caste differentiation, evolutionary constraints, morphological evolution, multivariate morphometrics, phylogenetic autocorrelation.

\section{Introduction}

The analysis of comparative data in evolutionary biology has, since Darwin's time, been the most commonly used technique for inferring adaptation and natural selection (Harvey \& Purvis, 1991). However, many recent developments in comparative data analysis have recognized that species do not usually provide independent points in a statistical analysis because they share characteristics through descent from common ancestors, at distinct levels (Felsenstein, 1985, 1988; Pagel \& Harvey, 1988; Bell, 1989; Garland et al., 1992; Gittleman \& Luh, 1992). It is therefore important to include phylogenetic information in statistical analysis of cross-species data to avoid spurious correlations suggesting adaptations.

Many statistical models have been developed to

${ }^{*}$ Correspondence. remove phylogenetic effects from cross-species data, permitting a more accurate analysis of adaptive trends (Felsenstein, 1985; Pagel \& Harvey, 1988; Bell, 1989; Garland et al., 1992, 1993; Gittleman \& Luh, 1992). One of these methods, clearly derived from quantitative genetics theory, is the phylogenetic autocorrelation analysis (Cheverud et al., 1985; Gittleman \& Kot, 1990), which starts by considering that the total variation $(T)$, for any quantitative character measured in related species, can be partitioned into a phylogenetic component $(P)$, which contains the variance shared by species, weighted by their phylogenetic distances, and a specific component $(S)$, which contains that part of the variation that is unique to each species, reflecting adaptations (modifications in a structure resulting from directional selection within lineages). These two components can be expressed as $T=P+S$, and an elevated $P$ value indicates that closely related species are similar for the character under study. This tendency, usually called 'phylogenetic inertia', can be explained 
by niche conservation, time lags and phenotypedependent responses to selection (Cheverud et al., 1985; Gittleman \& Kot, 1990; Harvey \& Purvis, 1991). This $P$ value can also be interpreted as a quantitative expression of the constraints limiting the power of natural selection to produce virtually any modification in organisms, and indicates how traits are controlled by complex epigenetic systems (expressed by a genetic covariance matrix) that arise as a product of long-term evolutionary history (Cheverud et al., 1985). On the other hand, adaptations must be inferred only from the $S$ values, because they indicate the part of the variation which is unique to each species, in such a way that correlations between $S$ values of distinct characters can indicate responses to selection in each species resulting from distinct levels of environmental variation and other selective pressures.

One of the striking aspects of eusocial behaviour in insects, especially in Hymenoptera, is caste differentiation resulting in a reproductive caste (one or more females, the queens, per colony) assisted by many sterile females (worker caste) (Wilson, 1971). Differences between castes are found in many morphological, behavioural and physiological characteristics and are obviously related to their roles in the social organization. In bees, body size variation between workers of distinct species has been primarily explained as an adaptation related to foraging activity and floral resource exploitation (Roubik \& Ackerman, 1987; Ruttner, 1988; Roubik, 1989; Baumgartner \& Roubik, 1989). This interpretation seems to be corroborated, for example, by differences between reproductive and worker castes across species of the genus Apis (Ruttner, 1988).

Stingless bees (Apidae: Meliponinae) are abundant, pantropical, highly eusocial insects that live in large perennial colonies (Wille, 1983). These bees have achieved their maximum diversity in the neotropics, both in terms of number of species (more than 300) (Roubik, 1989), and in morphological and behavioural characteristics such as nest building and resource exploitation patterns (Wille, 1983; Baumgartner \& Roubik, 1989). They also show a wide range of worker body size variation, ranging from small forms of Trigonini, such as species in the Plebeia-like group, to larger species of the genus Melipona (Wille, 1983). Consequently, a comparison of stingless bees, using phylogenetic autocorrelation analysis, may be useful to test the hypothesis of elevated residual effects on worker body size in relation to phylogenetic inertia, reflecting adaptations related to foraging activity and resource exploitation.

\section{Materials and methods}

We estimated worker body size in a multivariate sense by the scores of the first principal component derived from a covariance matrix of log-transformed characters (Neff \& Marcus, 1980). Eigenvectors were scaled to sum to 1.0 , and multivariate (evolutionary) allometric coefficients $k$ were estimated (Bookstein et al., 1985). Ten morphometric characteristics were measured on each individual worker bee (Table 1). Considering computational difficulties (related to eigenvalue extraction of nonsymmetric matrices, see below), we restricted our analyses to 16 species of stingless bees found in the neotropical region (Table 2). At least 10 individual bees of each species were measured.

Patterns of differentiation among species were described by a canonical variate analysis (CVA) (Neff \& Marcus, 1980; Bookstein et al., 1985), in an attempt to evaluate whether multivariate size is also the main component of variation among species. This was achieved by correlating the centroids of the first canonical axes with the first principal component derived from the covariance matrix of the log-transformed characters.

The scores of the mean vectors of each species on the first principal component (PC1) were then submitted to a phylogenetic autocorrelation analysis, following the general procedures described by Cheverud et al. (1985) and Gittleman \& Kot (1990). Initially, patterns of autocorrelation were evaluated using Moran's $I$ coefficient, given by

$I=\Sigma_{i} \Sigma_{j}\left(w i j Z_{i j}\right)$

where $w_{i j}$ are the elements of the symmetric matrix $\mathbf{W}$, which contains an expression of phylogenetic relationship between pairs of species (constrained in such a way that $\Sigma_{i} \Sigma_{j} w_{i j}=1.0$ ), and $\mathbf{Z}$ is a matrix of cross-products of the standardized vector of the character studied. This formulation of Moran's $I$ coefficient (Hubert et al., 1981) permits evaluation of its statistical significance by randomization approaches such as the Mantel test, and in this paper 1000 random permutations were used for each coefficient. Six coefficients were estimated in a phylogenetic correlogram, using connections $\mathbf{W}$ at distinct levels based on the cladograms provided in the recent revision by Michener (1990) (phylogenetic levels from 1 to 6 in Fig. 1). The significance of the correlogram as a whole was established using the Bonferroni criterion (Oden, 1984).

Because monotonic decay of phylogenetic autocorrelations was found across cladogram levels (a phylogenetic gradient), it is possible to remove $P$ 
Table 1 Characters analysed for 16 stingless bee species, their coefficients on PC1, multivariate allometric coefficients $(k)$ and Pearson correlations with phylogenetic $\left(r_{\mathrm{P}}\right)$ and specific $\left(r_{\mathrm{S}}\right)$ components

\begin{tabular}{lcccc}
\hline & & & \multicolumn{2}{c}{ Structure coefficients } \\
\cline { 4 - 5 } Character & PC1 & $k$ & $r_{\mathrm{P}}$ & $r_{\mathrm{S}}$ \\
\hline Glossa length & 0.480 & 1.493 & 0.576 & 0.754 \\
Mandible length & 0.344 & 1.070 & 0.706 & 0.705 \\
Mesoscutum width & 0.322 & 1.001 & 0.722 & 0.775 \\
Tibia length & 0.242 & 0.752 & 0.551 & 0.698 \\
Tibia width & 0.299 & 0.930 & 0.507 & 0.837 \\
Corbiculae length & 0.379 & 1.178 & 0.405 & 0.923 \\
Forewing length & 0.258 & 0.802 & 0.615 & 0.846 \\
Forewing width & 0.280 & 0.871 & 0.540 & 0.822 \\
Hindwing length & 0.306 & 0.951 & 0.606 & 0.814 \\
Hindwing width & 0.306 & 0.951 & 0.649 & 0.819 \\
\hline
\end{tabular}

Table 2 The sixteen stingless bee species analysed, followed by an identification code, and their total values for worker body size (scores on the first principal component - PC1) partitioned into phylogenetic $(P)$ and specific $(S)$ effects

\begin{tabular}{llrrr}
\hline Species & Code & \multicolumn{1}{c}{ PC1 } & \multicolumn{1}{c}{$P$} & \multicolumn{1}{c}{$S$} \\
\hline Frieseomelitta varia & FV & 0.111 & -0.089 & 0.200 \\
Frieseomelitta languida & FS & -0.338 & -0.022 & -0.316 \\
Geotrigona inusitata & GI & 0.039 & -0.073 & 0.113 \\
Cephalotrigona capitata & CC & 1.478 & -0.231 & 1.709 \\
Trigona hypogea & TH & -0.349 & -0.089 & -0.260 \\
Trigona recursa & TR & -0.190 & -0.116 & -0.074 \\
Tetragonisca angustula & TA & -1.211 & -0.073 & -1.138 \\
Plebeia droryana & PD & -1.063 & -0.364 & -0.699 \\
Plebeia poecilochroa & PP & -1.008 & -0.374 & -0.634 \\
Scaura latitarsis & SL & -1.304 & -0.312 & -0.992 \\
Partamona cupira & PC & 0.828 & -0.273 & 1.101 \\
Nannotrigona testaceicomis & NT & -0.869 & -0.052 & -0.817 \\
Scaptotrigona postica & SP & 0.255 & -0.193 & 0.448 \\
Scaptotrigona depilis & SD & 0.283 & -0.197 & 0.481 \\
Melipona scutellaris & MS & 1.841 & 1.100 & 0.741 \\
Melipona quadrifasciata & MQ & 1.496 & 1.359 & 0.137 \\
\hline
\end{tabular}

effects using an autoregressive model. Partition of $T$ into $P$ and $S$ effects can be performed by the linear model

$\mathbf{Y}=p \mathbf{W} \mathbf{Y}+\mathbf{E}$.

In this model, $\mathbf{Y}$ is the vector that describes body size variation in species (the first principal component) and $p$ is the phylogenetic autoregressive coefficient. The term $p \mathbf{W Y}$ corresponds to the phylogenetic component $P$ and $\mathbf{E}$ to the residual (specific) effect $S$. The matrix $\mathbf{W}$ in the autoregressive model describes the overall phylogenetic relationship between pairs of $n$ species (with zeros in the main diagonal), and it is standardized by rows to sum to 1.0 prior to the analysis. The elements of $\mathbf{W}$ were also defined based on the cladogram in Fig. 2 as

$W_{i j}=\left(L-l_{i j}\right) / L$,

where $l_{i j}$ is the level of connection between species $i$ and $j$ and $L$ is the total number of linkages in the cladogram (Lynch, 1991). This way, we assumed that branch lengths are equal, in the absence of more precise information about evolutionary time of 


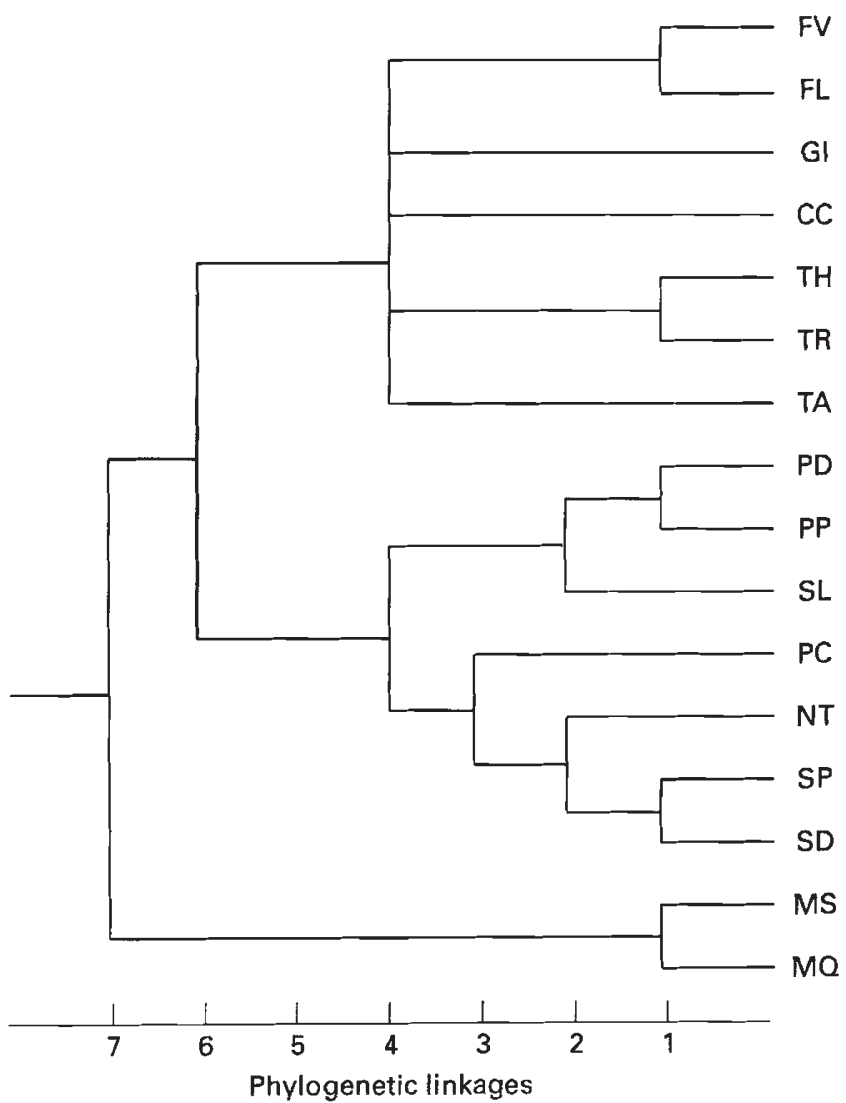

Fig. 1 Phylogenetic linkages among stingless bee species analysed, based on the cladogram of Michener (1990). divergence between species (Martins \& Garland, 1991). According to a recent study comparing these methods (Gittleman \& Luh, 1992), phylogenetic autocorrelations are less sensitive to errors in branch length information than other methods, such as the independent contrasts method of Felsenstein (1985).

The phylogenetic autoregressive coefficient $p$ cannot be estimated by an ordinary least squares regression, and for this reason a maximum likelihood equation was developed. The value of $m$ to be minimized to provide an unbiased estimate of $p$ is given by

$$
\begin{aligned}
m= & \ln \left(\mathbf{Y}^{\prime} \mathbf{Y}-2 p \mathbf{Y}^{\prime} \mathbf{W Y}+p^{2}(\mathbf{W Y})^{\prime}(\mathbf{W Y})\right) \\
& -2 / n \Sigma \ln \left(1-p \lambda_{i}\right),
\end{aligned}
$$

where $\lambda_{i}$ are the $n$ eigenvalues of the nonsymmetric matrix $\mathbf{W}$. A complete derivation of the above equation can be found in Cheverud et al. (1985) and Gittleman \& Kot (1990). Because of the row standardization, $\lambda_{i}$ varies between -1.0 and +1.0 . Using this equation, we searched for $p$ in the range -1.0 to +1.0 , in steps of 0.001 . The standard error of $p$ was estimated by a jackknife procedure (Sokal \& Rohlf, 1981), recalculating the phylogenetic autoregression 16 times, removing one species each time. Knowing the standard error allowed the null hypothesis that there were no phylogenetic patterns in the body size variation across species to be tested using Student's $t$-distribution. The amount of varia-

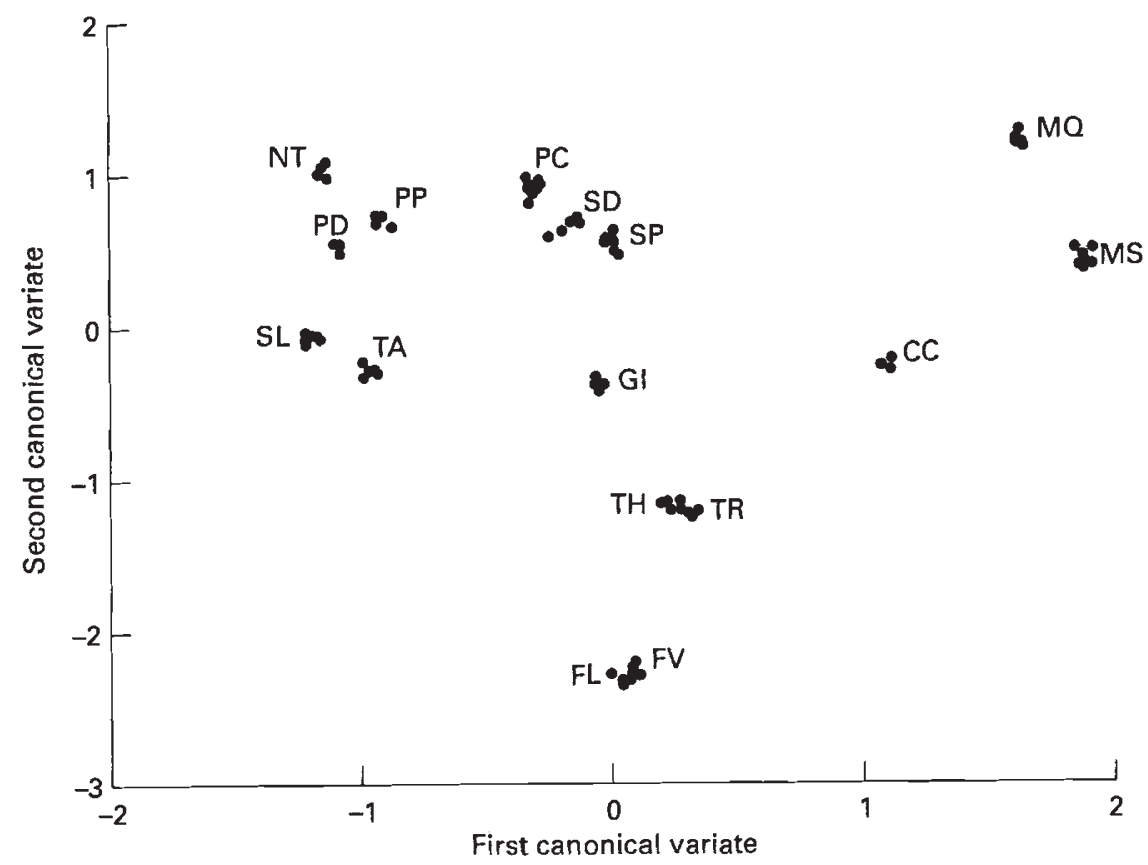

Fig. 2 Dispersion of individuals of the 16 stingless bee species in the space of the first two canonical variates. 
tion that can be attributed to residual (specific) effects is given as in a normal least squares regression by $S=1-p^{2}$.

\section{Results}

The first principal component accounts for 83.1 per cent of the morphometric variability among stingless bee species. The coefficients of 10 morphometric characters in this axis possess the same signal and have similar magnitudes (Table 1). Therefore, the first principal component can be interpreted as a general size factor (Neff \& Marcus, 1980; Bookstein et al., 1985). However, their coefficients are different from the expected value of $v^{-0.5}$ (where $v$ is the number of variables) for an isometric size vector, equal to 0.316 , indicating the existence of a large amount of evolutionary allometry (size-dependent shape variation). Thus, multivariate size must be interpreted as an allometric size vector, in which values of allometric coefficients $(k)$ different from 1.0 indicate nonlinear changes in characters (positive or negative) as size increases.

The dispersion of individuals analysed in the space of the first two canonical variates, which explain more than 99 per cent of the variability in the overall discriminant space, shows that most of the morphometric variation occurs among species (Fig. 2). More importantly, the first canonical variate can be also interpreted as an expression of multivariate body size, because there is a highly significant correlation of species centroids with principal component scores $(r=0.911 ; P<0.01)$.

Moran's $I$ coefficients of the phylogenetic correlogram of body size (Table 3) are significant at the 5 per cent level for the smallest and largest phylogenetic linkage levels. This indicates that phylogenetic autocorrelation in body size is caused by elevated similarity between closely related species associated with elevated dissimilarity between species in distinct tribes (Meliponini and Trigonini). The correlogram as a whole is significant according to the Bonferroni criterion, as at least one coefficient is significant at $P<0.0083(0.05 /$ number of linkages).

The estimated phylogenetic autoregressive coefficient $p$ was equal to $0.495 \pm 0.149$, significant at the 1 per cent level $\left(t_{15}=3.31\right)$. This indicates that 24.5 per cent of body size variation of stingless bee workers (the $p^{2}$ value), in a multivariate sense, can be attributed to phylogeny and evolutionary constraints. In order to verify that phylogenetic trends were in fact removed from the body size vector $T$ (first principal component) using the auto- regressive model, correlograms were also obtained for the $S$ values of each species (Gittleman \& Kot, 1990). All but one coefficient were not significant according to Mantel tests (Table 3), and using the Bonferroni criterion, the correlogram as a whole is not significant. This indicates that the autoregressive model was effective in removing phylogenetic trends. The relationships between each character and the $P$ and $S$ values were then obtained by structure coefficients (the Pearson product-moment correlation between partitioned principal component scores and each character across species), and are shown in Table 1. It is possible to see that all characters are more related to the specific component $S$, which is as expected considering the relatively greater effect of this component (75.5 per cent). The scores of species along the axis of $T$ (PC1), $P$ and $S$ values for multivariate body size are shown in Table 2 .

\section{Discussion}

The results of our work indicated a relatively low (but statistically significant) phylogenetic effect on worker body size variation in the neotropical stingless bees analysed, in such a way that interspecific variation of this composite trait is better explained by adaptations in each species, with small effects of 'inherited' patterns, or phylogenetic inertia. This corroborates the hypothesis of low evolutionary constraints and important adaptive trends related to resource exploitation in workers of highly eusocial bee species (Ruttner, 1988; Roubik, 1989). Also, this size axis contains a large amount of shape-dependent variation (evolutionary allometry), in such a way that adaptive trends involve both size and shape variation in morphological traits (Bookstein et al., 1985). This is very clear when considering that the structure coefficient on the $S$ component is highest

Table 3 Phylogenetic correlograms using Moran's I coefficients for scores of the first principal component $(T)$ and its specific effect $(S)$. In parentheses are the significance levels estimated for each coefficient by the Mantel test using 1000 random permutations

\begin{tabular}{lrr}
\hline Phylogenetic linkage level* $^{*} T(P)$ & \multicolumn{1}{c}{$S(P)$} \\
\hline 1 & $0.393(0.022)$ & $0.139(0.238)$ \\
2 & $0.221(0.114)$ & $0.110(0.283)$ \\
3 & $-0.021(0.474)$ & $0.008(0.471)$ \\
4 & $-0.759(0.252)$ & $-0.318(0.047)$ \\
5 & $-0.165(0.019)$ & $0.101(0.362)$ \\
6 & $-0.411(0.007)$ & $0.022(0.432)$ \\
\hline
\end{tabular}

*Levels illustrated in cladogram in Figure 1. 
for corbiculae length $(r=0.923)$, which is as expected because specific resource exploitation patterns have been widely used to explain morphological changes in this structure, as for example in necrophilous species of Trigona or cleptobiotic species of Lestrimelitta (Wille, 1983; Camargo \& Roubik, 1991). Our previous data also indicated a higher degree of additive genetic variance for morphometric traits and multivariate body size in at least one species of stingless bee (Scaptotrigona postica), in such a way that changes resulting from directional selection may occur in a relatively short period of time (Diniz-Filho \& Pignata, 1994).

In statistical terms, it is important to note that the phylogenetic autocorrelation coefficients are statistically significant. Further studies must take this into account to avoid spurious correlations when inferring Darwinian adaptations based purely on relationships between worker body size and components of environmental variation, such as size of exploited flowers or food-carrying capacity (Pagel \& Harvey, 1988; Gittleman \& Kot, 1990; Gittleman \& Luh, 1992).

The autocorrelation patterns found in the species analysed can be described by the phylogenetic correlogram as a monotonic decrease in the coefficients. This indicates a phylogenetic gradient in worker body size, in which morphological difference is proportional to cladistic distance (Gittleman \& Kot, 1990). The main problem with this interpretation of the phylogenetic gradient is the uncertainty about the phylogenetic connectivity matrix $\mathbf{W}$, because phylogenetic relationships are usually open to many interpretations (Cheverud et al., 1985; Pagel \& Harvey, 1988). In our case, the cladogram proposed by Michener (1990) for stingless bees has been criticized by Camargo \& Pedro (1992). We believe that these criticisms will not significantly affect our analyses, as the positive sign of the autoregressive coefficient and the observation of the phylogenetic correlograms in Table 3 indicated that phylogenetic effects are concentrated on the smallest and largest levels of species linkage. In other words, phylogenetic autocorrelation in body size is caused by increased similarity between closely related species, associated with increased dissimilarity between species in the two usually recognized tribes (Meliponini and Trigonini). At these two extreme levels taxonomic discussion is not very important, although Michener (1990) and Camargo \& Pedro (1992) discussed a possible phylogenetic link between the tribe Meliponini (genus Melipona) and the Plebeialike group of Trigonini. However, Michener (1990) finally considered Melipona to be the sister group to all other Meliponinae, but recognized no tribal distinction between Melipona and other genera. Because Melipona is larger than the other genera and was also considered here to be a sister group to them, the autoregressive coefficient estimated tends to be conservative (it can be smaller than it actually is). So, our study clearly supports the hypothesis of strong adaptive evolution of caste differentiation, permitting a more effective exploitation of resources by workers in highly eusocial bees.

\section{Acknowledgements}

We thank Drs W. E. Kerr and H. G. Fowler for many discussions about this project. Prof. M. A. P. Abuabara helped with matrix operations in the autoregressive model. This paper was partially supported by Conselho Nacional de Desenvolvimento Científico e Tecnológico ( $\mathrm{CNPq}$ ) of Brazil, through Doctoral and Research grants to the authors, and by the Pró-Reitoria de Pesquisa e Pós-Graduação (PRPPG/FUNAPE), Universidade Federal de Goiás, to J.A.F.D.-F.

\section{References}

BAUMGARTNER, D. AND ROUBiK, D. W. 1989. Ecology of necrophilous and filth-gathering stingless bees (Apidae, Meliponinae) of Peru. J. Kans. Entomol. Soc., 62, 11-22.

BELl, G. 1989. A comparative method. Am. Nat., 133, $553-571$.

BOOKSTEIN, F., CHERNOFF, B., ELDER, R. L., HUMPHRIES, J. M., SMITH, G. R. AND STRAUSS, R. E. 1985. Morphometrics in Evolutionary Biology. Academy of Natural Sciences of Philadelphia, special publication 15 .

CAMARGO, J. M. F. AND PEDRO, S. R. M. 1992. Systematics, phylogeny and biogeography of the Meliponinae (Hymenoptera, Apidae): a mini-review. Apidologie, 23, 509-522.

CAMARgo, J. M. F. AND RoubiK, D. w. 1991. Systematics and bionomics of the apoid obligate necrophages: the Trigona hypogea group (Hymenoptera: Apoidea; Meliponinae). Biol. J. Linn. Soc., 44, 13-39.

CHEVERUd, J. M., DOW, M. M. AND LEUTENEGGER, w. 1985. The quantitative assessment of phylogenetic constraints in comparative analyses: sexual dimorphism in body weight among primates. Evolution, 39, 1335-1351.

DiNiz-Filho, J. A. F. AND PignATA, M. 1. B. 1994. Quantitative genetics of multivariate morphometric variation in the Neotropical stingless bee, Scaptotrigona postica (Hymenoptera: Meliponinae). Braz. J, Genet., 17, 259-265.

FELsensteln, J. 1985. Phylogenies and the comparative method. Am. Nat., 125, 1-15.

FELSENStein, J. 1988. Phylogenies and quantitative characters. Ann. Rev. Ecol. Syst., 19, 445-471.

(C) The Genetical Society of Great Britain, Heredity, 76, 222-228. 
Garland, T., Harvey, P. H. AND IVES, A. R. 1992. Procedures for the analysis of comparative data using phylogenetically independent contrasts. Syst. Biol., 41, 18-32.

GARLAND, T., DICKERMAN, A. W., JANIS, C. M. AND JONES, J. A. 1993. Phylogenetic analysis of covariance by computer simulation. Syst. Biol., 42, 265-292.

GITTlEMAN, J. L. AND KOT, M. 1990. Adaptation: statistics and a null model for estimating phylogenetic effects. Syst. Zool., 39, 227-241.

GITTLEMAN, J. L. AND LUH, H.-K. 1992. On comparing comparative methods. Ann. Rev. Ecol. Syst., 23, 383-404.

HARVEY, P. H. AND PURVIS, A. 1991. Comparative methods for explaining adaptations. Nature, 351, 619-624.

HUBERT, L. J., GOLlEDGE, R. G. AND CONSTANZO, C. M. 1981. Generalized procedures for evaluating spatial autocorrelation. Geogr. Anal., 13, 224-233.

LYNCH, M. 1991. Methods for the analysis of comparative data in evolutionary biology. Evolution, 45, 1065-1080.

MARTINS, E. P. AND GARLAND, T. 1991. Phylogenetic analyses of the correlated evolution of continuous characters: a simulation study. Evolution, 45, 534-557.

MICHENER, C. D. 1990. Classification of the Apidae
(Hymenoptera). Univ. Kans. Sci. Bull., 54, 75-164 NEFF, N. A. AND MARCUS, L. F. 1980. A Survey of Multivariate Methods for Systematics. Privately published, New York.

ODEN, N. L. 1984. Assessing the significance of a spatial correlogram. Geogr. Anal., 16, 1-16.

PAGEL, M. D. AND HARVEY, P. H. 1988. Recent developments in the analysis of comparative data. Q. Rev. Biol., 63, 413-440.

Roubik, D. w. 1989. Ecology and Natural History of Tropical Bees. Cambridge University Press, Cambridge.

ROUBIK, D. W. AND ACKERMAN, J. D. 1987. Long-term ecology of euglossine orchid-bees in Panama. Oecologia, 73, 321-333.

RUTTNER, F. 1988. Biogeography and Taxonomy of Honeybees. Springer-Verlag, Berlin.

SOKAL, R. R. AND ROHLF, F. J. 1981. Biometry, 2nd edn. W. H. Freeman, New York.

Wille, A. 1983. Biology of stingless bees. Ann. Rev. Ent., 28, 41-64.

wilson, E. o. 1971. The Insect Societies. Harvard University Press, Cambridge, MA. 